\title{
Timely removal of exogenous cytokinin and the prevention of auxin transport from the shoot to the root affect the regeneration potential of Arabidopsis roots
}

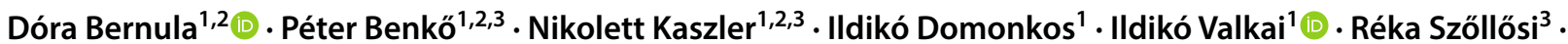 \\ Györgyi Ferenc $^{1}$ (D) Ferhan Ayaydin ${ }^{1}$ (i) $\cdot$ Attila Fehér ${ }^{1,3}$ (D) Katalin Gémes ${ }^{1,3}$ (i)
}

Received: 21 April 2019 / Accepted: 31 October 2019 / Published online: 8 November 2019

(c) The Author(s) 2019

\begin{abstract}
In vitro regeneration of Arabidopsis from roots is generally achieved via indirect organogenesis. First, transdifferentiation of lateral root primordia to calli is achieved by a balanced auxin-to-cytokinin ratio that is followed by the induction of shoot meristem formation using a high cytokinin level. Here we demonstrate that if the root explants were transferred onto a hormone-free medium after a transient (4-days) cytokinin treatment, embryogenic marker genes (LEC1, LEC2, FUS3) started to be expressed. App. 50\% of the regeneration foci developed into plantlets with trichome-less cotyledon-like leaves. Moreover, the somatic embryogenesis defective lecl mutant could regenerate only shoots with trichome-bearing leaves under this condition. Based on these observations, the mixed accomplishment of shoot organogenesis and somatic embryogenesis is hypothesized in the Arabidopsis root explants cultured under hormone-free conditions following cytokinin induction. Using whole seedlings instead of root explants in the same experimental set up, no regenerates were formed on the roots. Applying the auxin transport inhibitor TIBA to the root-to-shoot junction of the seedlings, the regeneration ability of the root could be restored. The observations indicate that shoot-derived endogenous auxin blocks the cytokinin-induced regeneration process in the roots of whole seedlings. The expression of the wound-induced transcription factor WIND1 could be detected in the roots of unwounded seedlings if the shoot-to-root auxin transport was inhibited. Manipulating the exogenous cytokinin level together with the endogenous shoot-to-root auxin transport therefore could mimic the effect of wounding (removal of shoot) on plant regeneration from roots.
\end{abstract}

\section{Key message}

Transferring root explants from high cytokinin to hormone-free conditions resulted in the expression of embryogenic markers. Inhibiting the shoot-to-root auxin transport had similar effect on regeneration as wounding.

Keywords Arabidopsis thaliana $\cdot$ Auxin transport inhibition $\cdot$ Cytokinin $\cdot$ Root explant $\cdot$ Shoot regeneration $\cdot$ Somatic embryogenesis · Wounding

Communicated by Danny Geelen.

Attila Fehér

feher.attila@brc.hu

Katalin Gémes

gemeskatalin80@gmail.com

1 Biological Research Centre, Institute of Plant Biology, 62, Temesvári krt, Szeged 6726, Hungary

2 Doctoral School of Biology, University of Szeged, 52, Közép fasor, Szeged 6726, Hungary

3 Department of Plant Biology, University of Szeged, 52, Közép fasor, Szeged 6726, Hungary

\section{Introduction}

In vitro plant regeneration techniques are widely used to preserve and propagate (clone) superior germplasms as well as to increase plant genetic variability via mutagenesis/transgenesis (Bhojwani and Dantu 2013). During the ca. hundred years of the history of plant cell and tissue culture (Sugiyama 2015), uncountable number of research articles reported the successful regeneration of various plant species, cultivars, explants. Two main systems are widely used for in vitro plant regeneration: de novo organogenesis or somatic 
embryogenesis (SE) (Ikeuchi et al. 2016). During de novo organogenesis, the establishment of apical meristems developing into shoots and roots, respectively, is induced successively. In contrast, embryo formation in somatic tissues during SE results in the autonomous development of whole plantlets in one step (Fehér et al. 2016). Both regeneration processes can occur either directly or indirectly without or with intermediate callus formation (Ikeuchi et al. 2016).

Auxin and cytokinin are considered as the two most significant plant hormones directly involved in plant regeneration but other plant hormones have also been implicated in the process (Su and Zhang 2014). It has been recognised more than 60 years ago that the ratio of exogenously applied auxin and cytokinin controls organ regeneration (Skoog and Miller 1957). While high auxin-to-cytokinin ratios promoted root development, high cytokinin-to-auxin ratios resulted in shoot regeneration on the explants from tobacco pith. High concentrations of both hormones resulted in overproliferation of explant cells and callus formation. This pioneering work was followed by the establishment of in vitro organogenesis systems in hundreds of plant species including the model plant Arabidopsis thaliana. Shoot regeneration on Arabidopsis explants is usually achieved in a two-step (indirect) strategy (Feldmann and Marks 1986; Valvekens et al. 1988): first, callus formation is induced using a balanced auxin and cytokinin ratio (callus induction medium; CIM) that is followed by shoot meristem formation due to a high cytokinin level (shoot induction medium; SIM).

Somatic embryogenesis can be induced by a variety of hormone and stress treatments depending on the species and/ or the type of explant. The most prevalent inducer is auxin, especially the artificial auxin 2,4-dichlorophenoxyacetic acid (2,4-D) (Fehér 2015). The effect of cytokinins on somatic embryogenesis is variable. In most systems, a low level of cytokinin is applied along with auxin to promote embryo induction while in other cases exogenous cytokinins were found to inhibit auxin-induced SE (see e.g. Mujib et al. 2016). In few systems, cytokinins were found to be effective as the sole inducers of embryo formation (e.g. Sagare et al. 2000; Chung et al. 2005; for review, Gaj 2004).

In addition to auxin and cytokinin, other plant hormones, such as ethylene and abscisic acid are also implicated in plant regeneration, especially in the stress-related induction of somatic embryogenesis (reviewed in, Su and Zhang 2014; Fehér 2015). Auxin and ethylene interacts during several regeneration processes (Su and Zhang 2014). Excessive ethylene produced due to ACC treatment generally inhibits regeneration. However, a transient increase in ethylene generation is hypothesized to be required for 2,4-D-induced somatic embryogenesis (Fehér 2015). In Medicago, it was shown that ethylene biosynthesis and the ethylene-response factor MtSERF1 are required for somatic embryogenesis (Mantiri et al. 2008). Abscisic acid is generally considered as antagonistic with callus formation and regeneration $(\mathrm{Su}$ and Zhang 2014). However, exogenous abscisic acid can also induce somatic embryo formation in specific explants, such as shoot tips of Arabidopsis (Kikuchi et al. 2006; Nishiwaki et al. 2000), and may play a role in the auxin-induced initiation of somatic embryogenesis (Su et al. 2013; Fehér 2015).

Although it is the ratio of exogenously applied plant hormones what one can easily manipulate to influence regeneration responses, it is the proper spatial distribution and temporal regulation of endogenous hormone levels that determine regeneration success (Jiménez 2005; Su and Zhang 2014). Among others, positive correlation was described between cytokinin levels and axillary bud outgrowth in various mutants and transgenic plants in which the cytokinin level was altered (Chaudhury et al. 1993; Catterou et al. 2002; Kakimoto 2001; Sun et al. 2003). Exogenously applied hormones and stress treatments also promote/inhibit regeneration at least partly via influencing endogenous hormone synthesis and transport (Jiménez 2005; Su and Zhang 2014; Fehér 2015). Bai et al. (2013) demonstrated that during 2,4-D-induced SE initiation in Arabidopsis calli several genes of cytokinin, abscisic acid, and ethylene synthesis and signalling were downregulated in parallel with the augmented expression of the YUCCA gene responsible for endogenous auxin synthesis.

Research on the model plant Arabidopsis thaliana has largely contributed to our understanding of many molecular and hormonal aspects of plant regeneration. Dynamic gradients of auxin and cytokinin responses were visualized using fluorescent reporters in indirect organogenesis and somatic embryogenesis of Arabidopsis (for review, Su and Zhang 2014). These gradients were in correlation with the de novo formation of shoot and/or root meristem organising centres. Spatiotemporal regulation of biosynthesis, transport, and degradation were found to be associated with the dynamics of hormone gradients (Cheng et al. 2013; Bai et al. 2013; $\mathrm{Su}$ and Zhang 2014). The role of polar auxin transport in the establishment of asymmetric hormone distribution is well demonstrated during the earliest phases of Arabidopsis regeneration ( $\mathrm{Su}$ et al. 2009, 2015). In agreement, the inhibition of auxin transport disrupts the spatiotemporal auxin distribution and prevents shoot regeneration (Cheng et al. 2013) as well as somatic embryogenesis (Elhiti and Stasolla 2011).

In addition to external hormones, wounding is also important in triggering the regenerative process ( $\mathrm{Xu}$ and Huang 2014). One of the key regulators, which is rapidly activated in response to wounding and has pivotal role in woundinduced callus formation, is the WOUND-INDUCED DEDIFFERENTIATION 1 (WIND1) transcription factor (Iwase et al. 2011a, b; 2015). Induced roots of unwounded Arabidopsis seedlings hardly exhibit indirect shoot organogenesis (Iwase et al. 2015). However, ectopic expression of WIND1 in uncut roots could bypass the requirement for 
wounding and auxin induction (Iwase et al. 2015). WIND1 overexpression did not lead to increased auxin accumulation in Arabidopsis, rather, WIND1 acted via a cytokinin signalling-dependent pathway to promote callus formation and shoot regeneration efficiency (Iwase et al. 2011a).

Auxin-induced initiation of direct somatic embryogenesis in Arabidopsis immature zygote explants is characterised by the expression of the seed-specific transcription factors LEAFY COTYLEDON 1 and 2 (LEC1 and LEC2) and FUSCA 3 (FUS3) (Gaj 2001; Gaj et al. 2005; Ledwoń and Gaj 2011). Mutants that are unable to express these genes fail to regenerate via the direct embryogenic pathway (Gaj et al. 2005) while transgenic seedlings ectopically expressing $L E C 1$ and $L E C 2$ produce somatic embryos on their cotyledons (Lotan et al. 1998; Stone et al. 2001). LEC2 activation resulted in embryogenic callus formation in 35S:LEC2-GR transgenic Arabidopsis roots in a wounding-dependent way (Iwase et al. 2015). However, exogenous hormone application on its own was not reported yet to induce embryogenic callus formation from Arabidopsis roots.

Here we report the evaluation of the regeneration potential of Arabidopsis roots applying a transient (4-days) high cytokinin treatment. Using the expression of the LEC1, LEC2, $F U S 3$ genes as embryogenesis markers, it was revealed that in this system the shoot organogenesis and somatic embryogenesis pathways were mixed. The cytokinin-treated Arabidopsis root explants expressed the above embryogenic markers under a subsequent hormone-free culture. Moreover, the lecl mutant exhibited significantly reduced regeneration efficiency indicating that in this culture regime part of the regeneration proceeds via somatic embryogenesis. Moreover, it could be established that shoot-derived auxin strongly inhibited the indirect shoot regeneration from the roots of cytokinin-induced unwounded seedlings, the regeneration potential of which could be restored with local auxin transport inhibitor (TIBA) application.

\section{Materials and methods}

\section{Plant materials and culture conditions}

Col-0 wild type and heterozygous leafy cotyledonl (lec1) mutant (AT1G21970) (Meinke et al. 1994) Arabidopsis thaliana seeds were provided by the Nottingham Arabidopsis Stock Centre (NASC) (Scholl et al. 2000). To produce wild type seedlings, seeds were surface-sterilized with $70 \%$ ethanol for $60 \mathrm{~s}$ followed by immersion in $4 \%$ commercial sodium hypochlorite solution (having $4.5 \%$ active chlorine) for $10 \mathrm{~min}$. The seeds then were rinsed for $1 \mathrm{~min}$ five times with sterile water. 90 seeds were placed in each $120 \times 120 \times 17 \mathrm{~mm}$ square plastic Petri dishes (Greiner Bio-One, International $\mathrm{GmbH}$, Kremsmuenster, Austria) containing full-strength
Gamborg B5 Medium Including Vitamins (Duchefa Biochemie B.V., Haarlem, The Netherlands,), $1 \%$ sucrose (VWR International LLC, Radnor, Pennsylvania, United States) and $1 \%$ plant agar (Duchefa Biochemie). The $\mathrm{pH}$ of the medium was adjusted to 5.7. Petri dishes were fixed vertically by special holders and placed in a growth chamber at $21{ }^{\circ} \mathrm{C}$, under continuous light with an irradiance of $50 \mu \mathrm{mol} \mathrm{m}^{-2} \mathrm{~s}^{-1}$ provided by white fluorescent tubes (Sylvania Luxline Plus; Feilo Sylvania Europe Limited, London, UK) for 1 week. Homozygous lecl seedlings were produced by culturing immature embryos on a medium containing half-strength Murashige and Skoog (MS) Medium Including Gamborg B5 Vitamins (Duchefa Biochemie), 1\% sucrose and 0.5\% plant agar. Germinated embryos exhibiting the lecl phenotype were selected and the seedlings were cultured under the same conditions as the wild type.

The in vitro shoot regeneration experiments were carried out under the same growth chamber conditions as described above. 7-days-old whole seedlings were placed onto a medium containing full-strength MS Basal Salt Mixture (Duchefa Biochemie), $2 \mathrm{ml} / \mathrm{l}$ vitamix [555 $\mathrm{mM}$ myoinositol (Duchefa Biochemie), $14.8 \mathrm{mM}$ thiamine- $\mathrm{HCl}$ (B1) (Duchefa Biochemie), $2.4 \mathrm{mM}$ pyridoxine- $\mathrm{HCl}$ (B6) (SERVA Electrophoresis GmbH, Heidelberg, Germany), $4.1 \mathrm{mM}$ nicotinic acid (B3) (Reanal, Budapest, Hungary), $13.3 \mathrm{mM}$ glycine (Molar Chemicals, Halásztelek, Hungary), $0.2 \mathrm{mM}$ biotin (Reanal)], 3\% sucrose, $0.8 \%$ plant agar and $2.7 \mu \mathrm{M}$ naphthaleneacetic acid (NAA) (Duchefa Biochemie) at $\mathrm{pH} 5.8$, and cultured for $24 \mathrm{~h}$ vertically. Subsequently, each plantlets were transferred for three days to a hormonefree (HF) MS basal medium supplemented with $2 \mathrm{ml} / 1$ vitamix, $3 \%$ sucrose and $0.8 \%$ plant agar (pH 5.8). To induce regeneration, roots were excised from the shoots with a scalpel under sterile conditions. Sixteen root explants were then cultured first on the ARM I medium (Márton and Browse 1991) including MS Basal Salt Mixture, $2 \mathrm{ml} / \mathrm{l}$ vitamix, 3\% sucrose, $0.8 \%$ plant agar and different growth regulators, including 17.1 $\mu \mathrm{M}$ indole-3-acetic acid (IAA) (Duchefa Biochemie), $0.68 \mu \mathrm{M}$ 2,4-dichlorophenoxyacetic acid (2,4D) (Sigma-Aldrich, St. Louis, Missouri, United States), $2.68 \mu \mathrm{M}$ 6-benzyladenine (BA) (Sigma-Aldrich), $1.48 \mu \mathrm{M}$ isopentenyl-adenine (IPA) (Sigma-Aldrich) for 3 days (if not indicated otherwise) then on ARM IIr medium (Márton and Browse 1991), containing MS Basal Salt Mixture, $2 \mathrm{ml} / \mathrm{l}$ vitamix, 3\% sucrose, $0.8 \%$ plant agar, $1.1 \mu \mathrm{M}$ NAA, $19.7 \mu \mathrm{M}$ IPA. The $\mathrm{pH}$ was adjusted to 5.8 for both media. After four days, eight root explants were transferred to hormone-free medium (MS salts, $2 \mathrm{ml} / 1$ vitamix, $3 \%$ sucrose, $0.8 \%$ plant agar). Non-dissected whole seedlings were cultured in the same way and in the same number as controls. To examine the effect of shoot-derived auxin on the regeneration process, $5 \mu \mathrm{M}$ auxin polar transport inhibitor 2,3,5-triiodobenzoic acid (TIBA) (Fluka, Chemie GmbH, Buchs, Switzerland) 
was applied in a low gelling agarose (Sigma-Aldrich) droplet at the junction of the shoot to the root. Three independent replicates were analysed in all experiments.

\section{Light microscopy}

The number of dark-green morphogenic foci on root explants/roots and the presence/absence of trichomes on the first leaves were determined using an Olympus SZX12 stereo dissection microscope (Olympus Corporation, Sindzsuku, Tokyo, Japan). For the bright field images, white LED light source (Photonic Optics, Vienna, Austria) was used. Photos were captured using an Olympus Camedia C7070 digital camera and the DScaler software (version 4.1.15).

\section{Scanning electron microscopy (SEM) at low-vacuum}

Samples were vacuum infiltrated and fixed with $100 \%$ methanol for $20 \mathrm{~min}$, dehydrated in $100 \%$ ethanol for $30 \mathrm{~min}$ and then left in fresh $100 \%$ ethanol for overnight. Next day, the samples were critical point dried, mounted on SEM stubs and observed in a JSM-7100F/LV scanning electron microscope (JEOL Ltd., Akishima, Tokyo, Japan). The uncoated leaves and embryos were imaged by detecting backscattered electrons at $15 \mathrm{kV}$ accelerating voltage and $35 \mathrm{~Pa}$ pressure in the specimen chamber.

\section{Gene expression analysis}

Total RNA was extracted using Quick-RNA Miniprep Kit (Zymo Research, Irvine, California, United States) which includes the removal of contaminating genomic DNA. 500 to $1000 \mathrm{ng}$ of total RNA was reverse-transcribed for $20 \mathrm{~min}$ at $42{ }^{\circ} \mathrm{C}$ and for $10 \mathrm{~min}$ at $75{ }^{\circ} \mathrm{C}$ in a $20 \mu \mathrm{l}$ reaction volume using Precision nanoScript 2 RT kit (PrimerDesign Ltd., Chandler's Ford, United Kingdom) according to the manufacturer's instructions. cDNA products were diluted 1:10 in AccuGENE® water (Lonza, Verviers, Belgium). The expression of LEC1 (AT1G21970), LEC2 (AT1G28300), FUS3 (AT3G26790) and WIND1 (AT1G78080) genes was analysed by real-time quantitative PCR (qPCR). The expression of the AT2G41960 gene was used as reference. This gene was selected using the Arabidopsis Regeneration eFP browser at The Bio-Analytic Resource for Plant Biology (bar.u-toronto.ca; Winter et al. 2007) allowing the in silico analysis of gene expression based on transcriptomic data sets of root-to-shoot regeneration experiments (Chatfield et al. 2013). According to these data, the AT2G41960 gene has a constitutive expression during the process.

The RT-qPCR reactions were carried out by the Applied Biosystems ABI PRISM 7900HT Fast Real-Time PCR system (Thermo Fisher Scientific, Waltham, Massachusetts, United States) and the primers listed in Table 1 were used.
The PCR mixture contained (in a total volume of $14 \mu \mathrm{l}$ ) $6 \mu \mathrm{l}$ cDNA, $0.5 \mu \mathrm{l}$ forward primer, $0.5 \mu \mathrm{l}$ reverse primer, $7 \mu \mathrm{l}$ PrecisionPLUS MasterMix with ROX premixed with SYBRGreen (PrimerDesign Ltd.). For amplification, a standard two-step thermal cycling profile was used $\left(15 \mathrm{~s}\right.$ at $95^{\circ} \mathrm{C}$ and $1 \mathrm{~min}$ at $60^{\circ} \mathrm{C}$ ) during 40 cycles, after a $10 \mathrm{~min}$ preheating step at $95{ }^{\circ} \mathrm{C}$. Finally, a dissociation stage was added with $95^{\circ} \mathrm{C}$ for $15 \mathrm{~s}, 60^{\circ} \mathrm{C}$ for $15 \mathrm{~s}$ and $95^{\circ} \mathrm{C}$ for $15 \mathrm{~s}$. Data analysis was performed using SDS 2.3 software (Life Technologies, Carlsbad, California, United States). $\mathrm{C}_{\mathrm{T}}$ values were analysed using the RQ Manager Software version 1.2 (Life Technologies) and then exported to Microsoft Excel 2010 for further analysis. The ratio of each mRNA relative to the AT2G41960 was calculated using the (2) ${ }^{-\Delta \Delta C t}$ method. Data were averaged from three independent biological experiments with three technical replicates each.

\section{Statistical analysis and data representation}

Statistical evaluations were performed using the SigmaPlot v.12.0 statistical software. Quantitative data are presented as the mean $\pm \mathrm{SE}$ and the significance of difference between sets of data was determined by one-way analysis of variance (ANOVA) following Duncan's multiple range tests; a $P$ value of less than 0.05 was considered significant.

\section{Results}

\section{Timely removal of cytokinin alters the regeneration pathway of Arabidopsis root explants}

The length of the period of cytokinin action required for the induction of shoot regeneration from Arabidopsis root explants was investigated. It was found that approximately

Table 1 Sequences of the oligonucleotide primers used in the qPCR experiments

\begin{tabular}{ll}
\hline Primer sequences & Gene \\
\hline F: 5'-GTTATGGTATGTTGGACC & AtLEC1 (AT1G21970) \\
AATCC-3' & \\
R: 5'-TTCATCTTGACCCGACGAC-3' & \\
F: 5'-TGATACTCCCGAAGAAAGCC-3' & AtFUS3 (AT3G26790) \\
R: 5'-CTATACTTGAAGGTCCAA & \\
ACGTG-3' & \\
F: 5'-CGCCATGAATGGAAATTCGG-3' & AtLEC2 (AT1G28300) \\
R: 5'-CCACGTACGCGTGATCCTCA-3' & \\
F: 5'-TGGCGGAGACTCAGAAACAG-3' & AtWIND1 (AT1G78080) \\
R: 5'-GGCGACGAAACCTTCTTCTCA-3' & \\
F: 5'-TTCAATTAAAAGAGGGGAATGC- & AT2G41960 \\
3' & \\
R: 5'-CTTTGTCTATCAGGACTTCAT & \\
CCTC-3' & \\
\hline
\end{tabular}




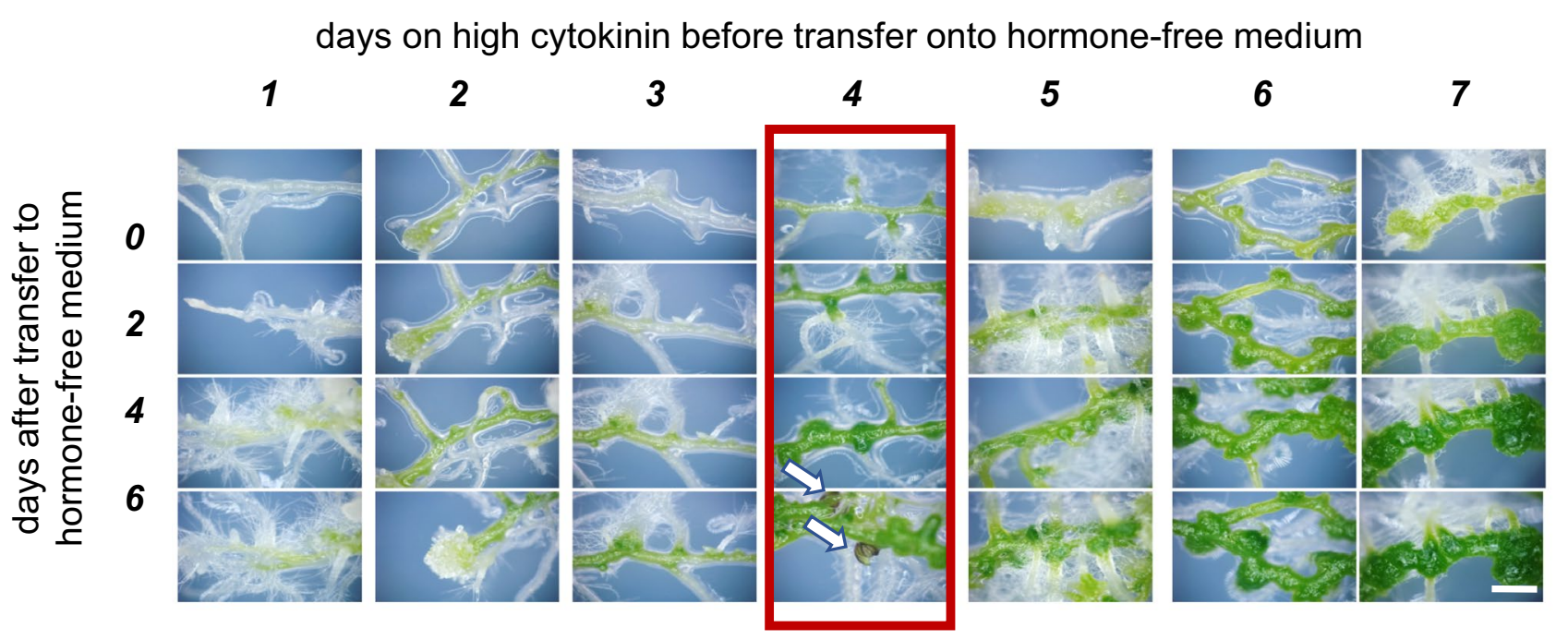

Fig. 1 Callus formation and plant regeneration on root explants cultured for the indicated periods (1-7 days) on the high cytokinin lowauxin shoot induction medium (ARM IIr) before transferring them to hormone-free conditions. Pictures were taken $0,2,4$ or 6 days after

4 days were required on the high cytokinin medium to efficiently induce morphogenic foci on the root explants (Fig. 1). Interestingly, transferring the root segments to hormone-free conditions after 4 days of cytokinin induction, callus growth was restricted, and green globular structures appeared on the explants some of which developed into somatic embryo-like structures following ca. 6 more days of incubation (Figs. 1, 2a, b). Additional 2 weeks on the hormone-free medium resulted in plantlet regeneration from these structures (Fig. 2c). Approximately half of the regenerated plantlets had trichome-less first leaves resembling cotyledons (Fig. 2c, d; Table 2). The absence of trichomes is often used as a morphological marker for somatic embryo formation since somatic embryos develop cotyledon-like trichome-less first leaves (e.g. Horstman et al. 2017).

Keeping the root explants continuously on the high cytokinin medium, however, we found, extensive callus formation (Fig. 2e, f). Culturing the explants for further 2 weeks on this medium, shoots appeared on the surface of explants. The regenerates had trichome-bearing first leaves (Fig. 2g, h) indicating their formation via shoot organogenesis and not somatic embryogenesis.

To verify whether the developmental pathway leading to trichome-less regenerates is indeed somatic embryogenesis, the relative expression of three embryogenesis-associated genes (LEC1, LEC2, and FUS3) was determined (Fig. 3). the transfer to the hormone-free medium. White arrows point to the regenerated somatic embryo-like structures on roots cultured for 4 days on the high cytokinin medium (red box) followed by 6 days on the hormone-free one. The scale bar is $1 \mathrm{~mm}$. (Color figure online)

It was established that culturing the cytokinin-induced root explants on hormone-free medium for 5 days resulted in the increased expression of all three investigated genes. The expression of the genes was not augmented in cultures maintained continuously on the cytokinin-rich medium strengthening that shoot organogenesis rather than somatic embryogenesis was induced under that condition (Fig. 3). To further support the view that the transient cytokinin treatment indeed led to somatic embryogenesis, root explants of the embryogenesis pathway defective lecl mutant was subjected to the same culture regime (Fig. 4; Table 2). It was found that culturing root explants continuously on the cytokinin-containing medium, the mutant reacted on the same way as the wild type (Fig. 4a, b). It formed green calli and subsequently shoots. However, if lecl root segments were moved to hormone-free medium after 4 days of cytokinin induction, the development of calli was restricted and the number of potentially morphogenic green foci was approximately the half as compared to the wild type (Fig. 4c, d; Table 2). From the lecl roots, only shoots with trichomebearing leaves could be regenerated (Table 2). Altogether, the above observations indicated that timely removal of cytokinin can result in the parallel/mixed development of shoots and somatic embryo-like structures while the latter is prevented by the continuous presence of high concentration of cytokinin in the medium. The somatic embryo-like 
Fig. 2 Plant regeneration of root explants after moving them to hormone-free conditions following a period of 4 days on the high cytokinin medium (a-d) or in the continuous presence of high cytokinin concentration (e-h). Pictures of root explants $(\mathbf{a}, \mathbf{e})$ and their morphogenic regions $(\mathbf{b}, \mathbf{f})$ were taken 5 days after the transfer to hormonefree medium following the previous period of 4 days on the high-cytokinin low-auxin ARM IIr medium $(\mathbf{a}, \mathbf{b})$ or 9 days after the transfer to the ARM IIr medium (e, f). The first leaves of the regenerated plantlets were investigated 2 weeks later (c d, g, h). Light microscopy (a, $\mathbf{c}, \mathbf{e}, \mathbf{g})$ and scanning electron microscopy $(\mathbf{b}, \mathbf{d}, \mathbf{f}, \mathbf{h})$ were used to take the images. The bars represent $1 \mathrm{~mm}(\mathbf{a}, \mathbf{c}, \mathbf{e}$, g), and $100 \mu \mathrm{m}(\mathbf{b}, \mathbf{d}, \mathbf{f}, \mathbf{h})$, respectively
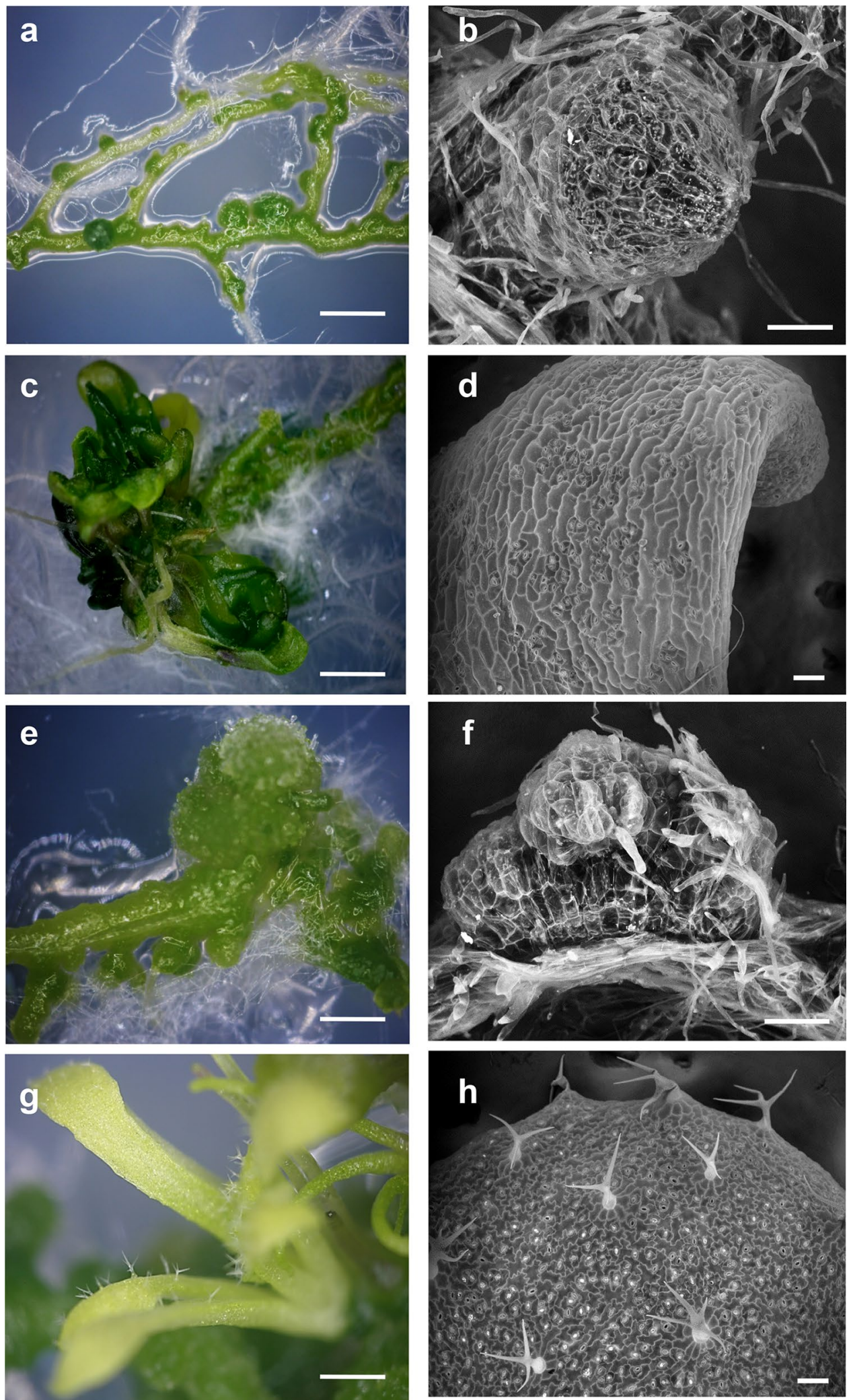
Table 2 Regeneration efficiency and type of wild type (Col) and lecl mutant seedling roots/root explants with or without TIBA treatment

\begin{tabular}{lccccc}
\hline Plant material & TIBA & $\begin{array}{l}\text { No. of regenera- } \\
\text { tion foci/root }\end{array}$ & $\begin{array}{l}\text { \% of regenerated } \\
\text { plantlets }^{\mathrm{b}}\end{array}$ & $\begin{array}{l}\text { \% of trichome- } \\
\text { less plantlets }\end{array}$ & $\begin{array}{l}\text { \% of trichome- } \\
\text { bearing plantlets }\end{array}$ \\
\hline Col root explant & - & $8 \pm 0.8$ & $65.6 \pm 3.7$ & $56.9 \pm 4.7$ & $43.1 \pm 4.7$ \\
lec1 root explant & - & $3.9 \pm 0.4$ & $23.8 \pm 6.9$ & $0.0 \pm 0.0$ & $100 \pm 0.0$ \\
Col seedling & - & $0.0 \pm 0.0$ & $0.0 \pm 0.0$ & $0.0 \pm 0.0$ & $0.0 \pm 0.0$ \\
Col seedling & + & $6.8 \pm 0.8$ & $43.8 \pm 4.5$ & $48.3 \pm 3.9$ & $51.7 \pm 3.9$ \\
lec1 seedling & - & $0.0 \pm 0.0$ & $0.0 \pm 0.0$ & $0.0 \pm 0.0$ & $0.0 \pm 0.0$ \\
lec1 seedling & + & $3.3 \pm 0.4$ & $15.0 \pm 5.1$ & $0.0 \pm 0.0$ & $50.0 \pm 15.7$ \\
\hline
\end{tabular}

${ }^{\mathrm{a}}$ Green morphogenic foci per root explant (counted after 5 days following transfer to hormone-free medium). Average and standard error

${ }^{b}$ Percentage of the potential morphogenic foci regenerated into normal plantlets per explant. Average and standard error

${ }^{c}$ Percentage of regenerated plantlets having leaves/cotyledons with/without trichomes per explant. Average and standard error structures had no clear bipolar structure, indicating that root morphogenesis was inhibited, e.g. due to the remained action of the high cytokinin concentration in the previous medium.

\section{Shoot-derived auxin prevents the regeneration from the roots of Arabidopsis seedlings}

Interestingly, the above described regeneration system was inefficient, and no regenerates could be observed when whole seedlings were used instead of root explants (Fig. 5; Table 2). Roots of the whole seedlings that were cultured on high cytokinin medium thickened and became green but failed to regenerate calli and shoots (Fig. 5a; Table 2). When the seedlings were removed to hormone-free medium after 4 days of the cytokinin treatment, the thickening and greening of the root did not take place (Fig. 5b). The potential role of shoot-derived auxin on the regeneration potential of the root was tested using the auxin transport inhibitor TIBA. $5 \mu \mathrm{M}$ TIBA in a low gelling temperature agarose drop was applied to the shoot-to-root junction of seedlings before they were transferred to the high cytokinin medium. TIBA application restored the regeneration potential of the seedling roots (Fig. 5c, d; Table 2). Like the excised root explants (Fig. 5e, f), the roots of TIBA-treated seedlings regenerated only trichome-bearing shoots on the high cytokinin medium. Approximately $50 \%$ of regenerated shoots were devoid of trichomes in case of transient (4 days) cytokinin treatment followed by hormone-free culture. The trichome-less plantlets are likely the result of somatic embryogenesis. It is supported by the increased relative expression of the three embryogenesis markers in these roots (Fig. 6). Moreover, the regeneration of TIBA-treated lecl seedlings did not result in trichome-less plantlets (Table 2).

Wounding and the wound-induced expression of the WIND1 transcription factor are considered to have a central role in the increased regeneration potential of root explants as compared to the roots of intact seedlings (Iwase et al. 2015). Therefore, the expression of WIND1 in the roots of TIBA-treated seedlings was investigated. Similar to wounding, blocking the shoot-to-root auxin transport by a TIBA-containing agarose droplet resulted in increased WIND1 expression in the root (Fig. 6) in agreement with the increased regeneration potential.

\section{Discussion}

Exogeneous application of cytokinins (with or without auxin) is widely used to induce de novo axillary shoot meristem formation in vitro. This agrees with observations that mutations or transgenes elevating the endogenous cytokinin level promote shoot formation (Chaudhury et al. 1993; Catterou et al. 2002; Kakimoto 2001; Sun et al. 2003) while in mutants with reduced cytokinin synthesis this process is prevented (Cheng et al. 2013).

We observed that the expression of embryogenic markers could be achieved by manipulating the time spent by Arabidopsis root segments on high-cytokinin and low-auxin (19.7 $\mu \mathrm{M}$ IPA and 1.1 $\mu \mathrm{M}$ NAA) shoot induction medium transferring the explants into hormone-free conditions following a four-day-long incubation period (Fig. 3). Under these conditions, nearly half of the regenerated plantlets exhibited trichome-less cotyledon-like first leaves 

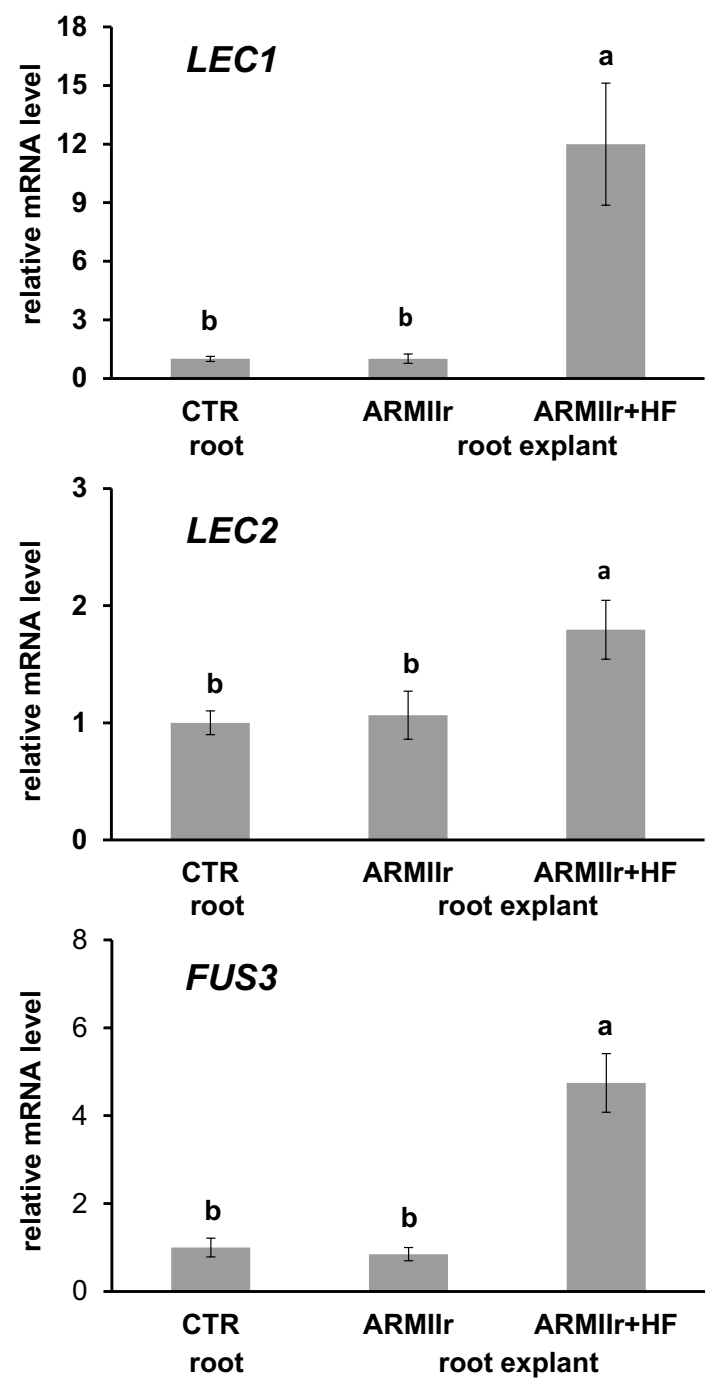

Fig. 3 Real-time quantitative PCR analysis of embryogenic gene expression (LEC1, LEC2 and FUS3) in untreated 7-days-old control seedling roots as reference (CTR root), in root explants at the time of 9 days on the high cytokinin ARM IIr medium (ARM IIr) and in root explants cultured 4 days on the ARM IIr medium followed by a hormone-free culture for 5 days (ARM IIr $+\mathrm{HF}$ ). The expression of the AT2G41960 gene was used for data normalization (see "Materials and methods" for details). Standard errors are shown on the columns. The significance of difference between sets of data was determined by one-way analysis of variance (ANOVA) following Duncan's multiple range tests; a $P$ value of less than 0.05 was considered significant as indicated by different letters strengthening the view that they were formed via an embryogenic pathway (Fig. 2; Table 2). Under the same conditions, the somatic embryogenesis-defective lecl mutant roots could regenerate only shoots with trichome-bearing true first leaves (Fig. 4; Table 2). The number of lecl regenerants was about the half that of the wild type (Table 2). These observations indicated that the shoot regeneration and the embryogenesis pathways were induced in parallel and only the latter was blocked by the continuous presence of the high concentration of cytokinin. The absence of trichomes on the first leaves is often used as a morphological marker for somatic embryo formation but this marker can be misleading as it can be delayed initially (Horstman et al. 2017). However, in our experiments, regenerants with trichomeless leaves appeared only in conditions when the embryogenic marker genes were also induced, but not if the plant was a non-embryogenic mutant (lecl). This made us confident that about half of the regenerants used at least initially an embryogenic pathway. Parallel induction of the two regeneration pathways (shoot organogenesis and somatic embryogenesis) has been described in Solanum carolinense explants exposed to low concentration of benzyladenine while only shoot regeneration was observed at higher concentrations (Reynolds 1986). Switching from organogenesis to somatic embryogenesis provoked by high sugar concentration in sunflower tissue culture was partly ascribed to reduced cytokinin uptake from the medium (Charrière and Hahne 1998). These observations could be explained with the inhibitory effect of cytokinin on SE. Cytokinin was reported to repress the expression of the transcription factors LEC2 and FUS3 (Casson et al. 2006) having key roles in somatic embryogenesis (Horstman et al. 2017). In agreement, moving the cytokinin-induced (for ca. 4 days) Arabidopsis root explants to hormone-free medium increased the expression of the embryogenesis regulators $L E C 1, L E C 2$, and FUS3 (Fig. 3).

One can hypothesize that indirect shoot organogenesis and indirect somatic embryogenesis share the same initial steps at least as the formation of the shoot meristem is concerned (Su et al. 2009, 2015; Cheng et al. 2013; Su and Zhang 2014). The multicellular origin of shoot primordia and somatic embryos from the same type of cells has been demonstrated in several systems further supporting the similarity of the initial steps of the two pathways (Bronner et al. 
Fig. 4 Plant regeneration of the embryogenesis defective lec1 mutant (a, c) and wild type (wt) (b, d) root explants in the continuous presence of high cytokinin concentration $(\mathbf{a}, \mathbf{b})$ or after moving them to hormone-free conditions following a period of 4 days on the high cytokinin medium $(\mathbf{c}, \mathbf{d})$. Pictures of root explants were taken 9 days after the transfer to the high cytokinin ARM IIr medium $(\mathbf{a}, \mathbf{b})$ and 5 days after the transfer to hormone-free medium following the previous period of 4 days on the ARM IIr medium (c, d), respectively. The scale bars represent $1 \mathrm{~mm}$
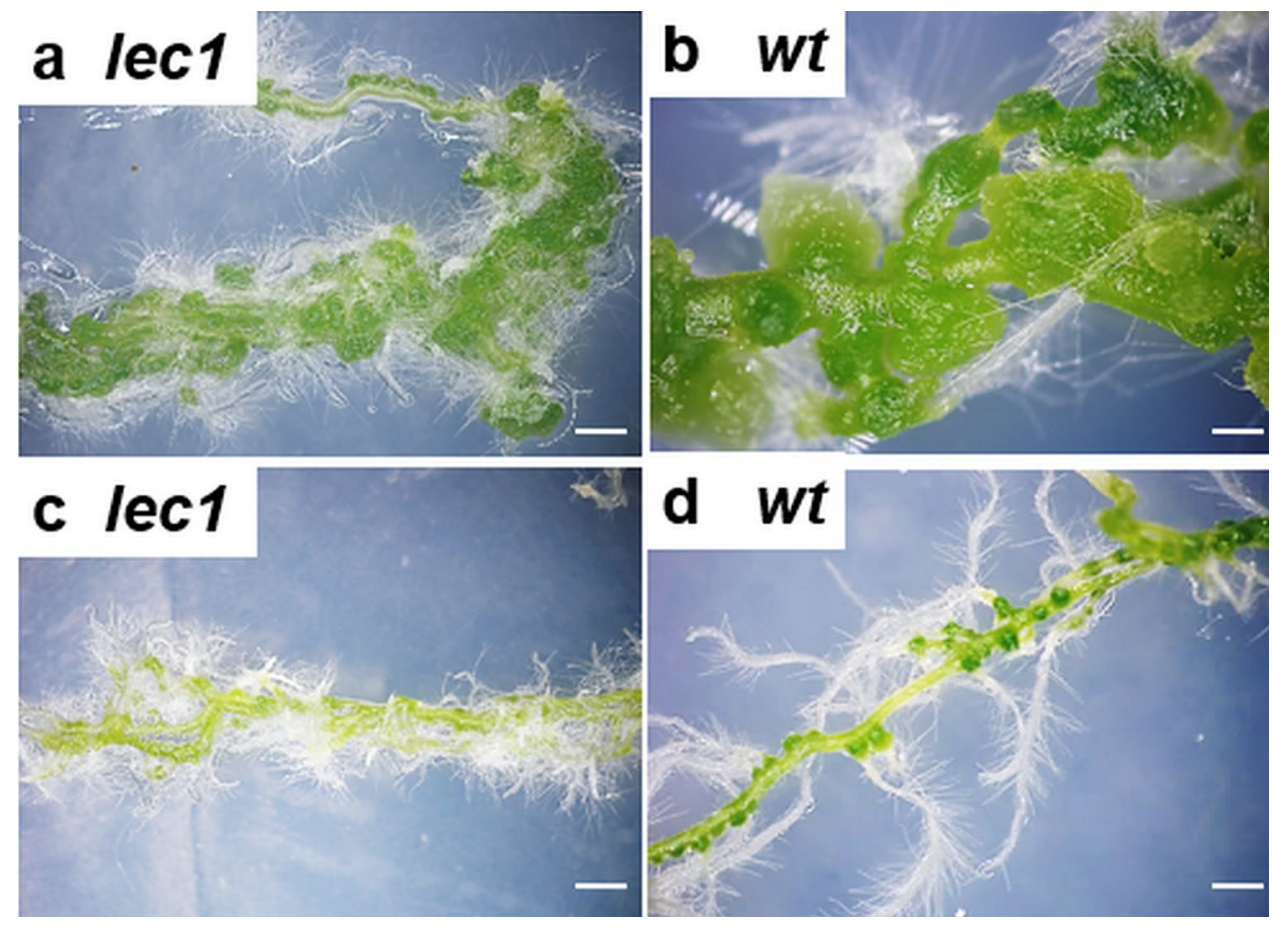

1994; Yumbla-Orbes et al. 2017). Timely manipulation of exogenous/endogenous hormone levels may allow the parallel manifestation of both pathways, in different but even in the same regeneration foci.

Removal of the high exogenous cytokinin concentration might induced a switch in the Arabidopsis root explants affecting endogenous cytokinin or auxin synthesis and/or transport. It has been demonstrated in many developmental systems that cytokinin and auxin mutually regulate each other's level, among others, including the inhibition of polar auxin transport by cytokinin (Pernisová et al. 2011; Schaller et al. 2015). The similar effect of high endogenous cytokinin and auxin transport inhibition, respectively, on in vitro plant regeneration was demonstrated using sunflower (Helianthus annuus L.) immature zygotic embryo explants. These explants regenerate shoots at $3 \%$ and somatic embryos at $12 \%$ sucrose concentration in a cytokinin-containing induction medium (Bronner et al. 1994). It was shown that the increased sucrose concentration resulted in lowered cytokinin uptake and an increased endogenous auxin/cytokinin ratio in association with the embryogenic response (Charrière et al. 1999). Inclusion of various auxin transport inhibitors to the high-sucrose induction medium prevented somatic embryogenesis but allowed shoot morphogenesis
(Charrière and Hahne 1998) mimicking the effect of the lower exogenous sucrose and higher endogenous cytokinin concentrations.

The inclusion of polar auxin transport inhibitors in the induction media prevented somatic embryogenesis in most of the cases or led to morphogenic abnormalities (e.g. Schiavone and Cooke 1987; Grzyb et al. 2018) although in special cases could promote the process at low concentrations (Chen and Chang 2004). Here we report that applying only a drop of the auxin transport inhibitor TIBA onto the shootto-root junction (an approach widely used to study the effect of shoot-derived auxin on root functions; e.g. Reed et al. 1998; Guo et al. 2005) promoted plant regeneration from the roots of unwounded Arabidopsis seedlings that are otherwise unresponsive to induction.

An interesting feature of indirect shoot regeneration from Arabidopsis roots that it is efficient only from excised explants but not in whole seedlings (Iwase et al. 2015) (Fig. 5a, b). In contrast, the direct conversion of lateral root primordia to shoot meristems in response to high exogenous cytokinin concentration can take place in whole seedling roots (Chatfield et al. 2013; Rosspopoff et al. 2017). Furthermore, mutant or transgenic plants with high endogenous cytokinin concentration exhibit increased shoot regeneration 
Fig. 5 Plant regeneration of the roots of whole seedlings (a-d) and root explants as controls $(\mathbf{e}, \mathbf{f})$ cultured in the continuous presence of high cytokinin concentration $(\mathbf{a}, \mathbf{c}, \mathbf{e})$ or after moving them to hormone-free conditions following a period of 4 days on the high cytokinin medium (b, d, f). In the case of whole seedlings, some of them was treated by applying $5 \mu \mathrm{M}$ TIBA in an agarose droplet to the shoot-root junction (c, d). Pictures were taken 9 days after the transfer to the high cytokinin ARM IIr medium (a, c, e) and 5 days after the transfer to hormone-free medium following the previous period of 4 days on the ARM IIr medium (b, d, f), respectively. The bars represent $1 \mathrm{~mm}$
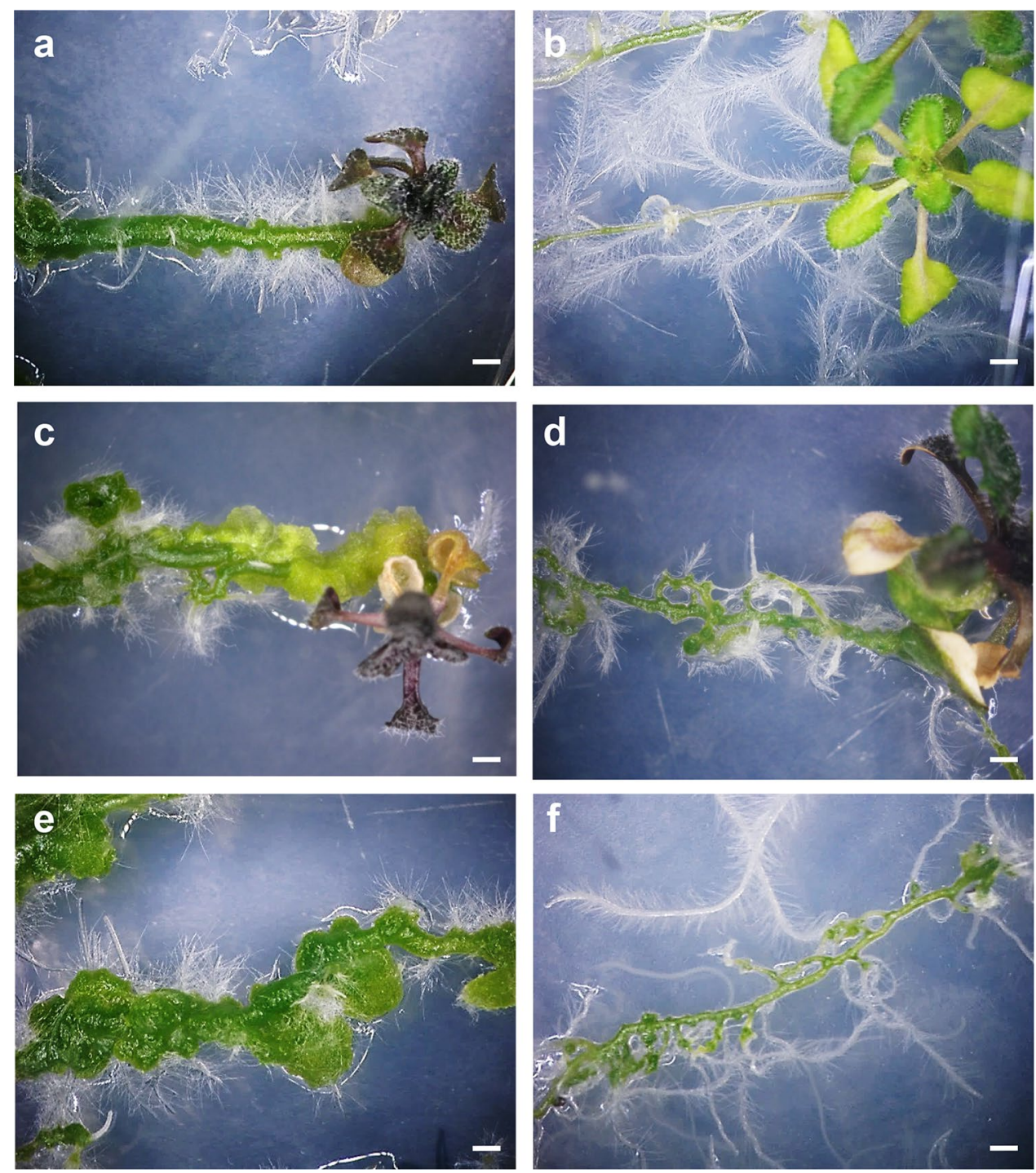

only on in vitro cultured root explants, but not on the roots of whole seedlings (Chaudhury et al. 1993; Catterou et al. 2002; Kakimoto 2001; Sun et al. 2003). Wounding was shown to promote regeneration-competent callus development from Arabidopsis roots in a WIND1 transcription factor-dependent way (Iwase et al. 2011a, b; 2015; Ikeuchi et al. 2016). Endogenous auxin level is not significantly changed in wounded Arabidopsis hypocotyls (Iwase et al. 2017) and WINDI has no effect on endogenous auxin level (Ikeda and Ohme-Takagi 2014; Iwase et al. 2017) but was shown to activate cytokinin synthesis and responses (Iwase et al. 2017).

Here we show that the effect of wounding on cytokininmediated shoot/embryo regeneration from roots could be mimicked blocking the auxin transport from the shoot to the root (Fig. 5; Table 2). Local auxin transport inhibitor (TIBA) application to the shoot-to-root junction resulted in increased WIND1 expression in the unwounded roots of seedlings (Fig. 6) in association with their increased shoot/embryo regeneration potential (Fig. 5; Table 2). Our observation indicates that either the removal of the shoot as the auxin source or blocking the shoot-to-root auxin transport result in increased WIND1 expression and enhanced competence for callus/shoot/embryo regeneration under appropriate inductive conditions. Shoot-derived auxin somehow interfere with this process. Polar auxin transport from the shoot to root is known to be required for lateral root formation (Reed et al. 

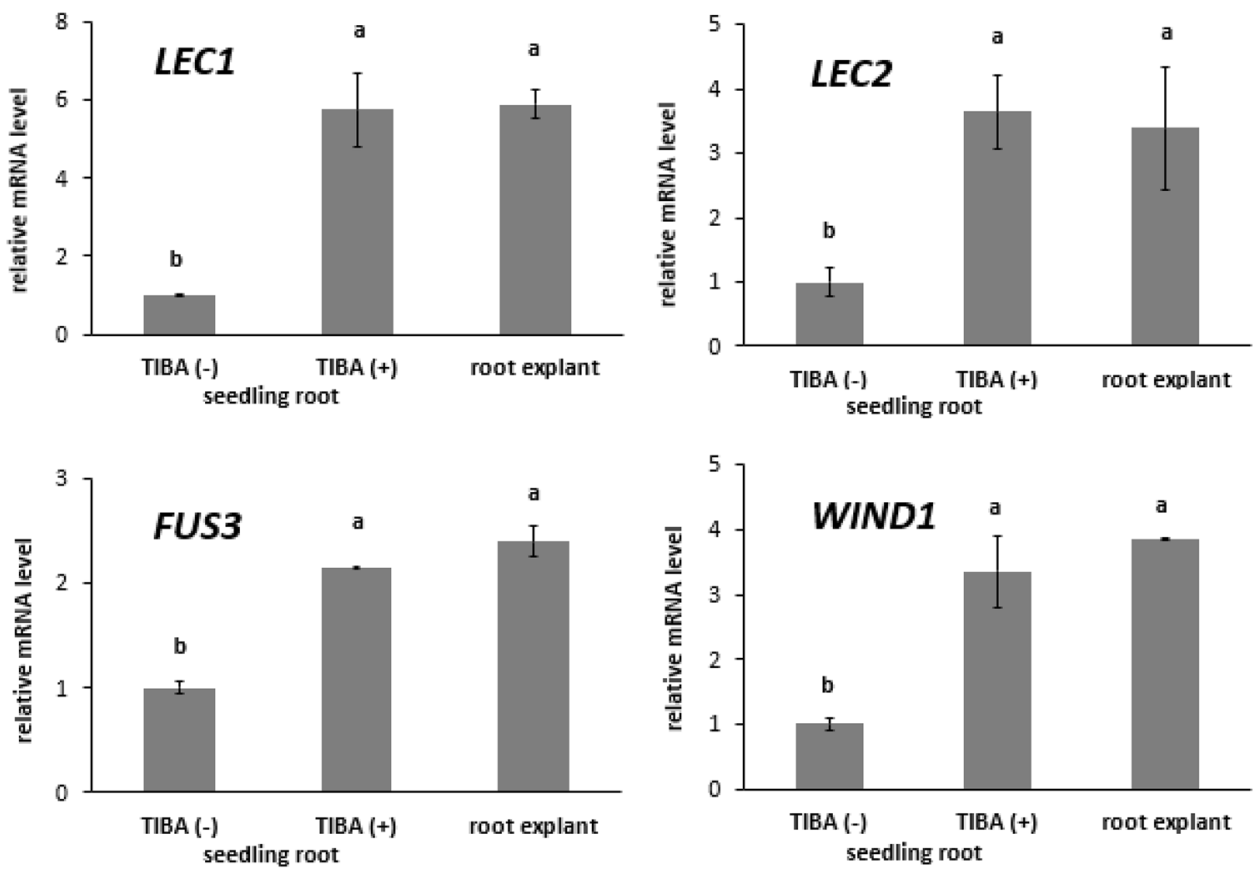

Fig. 6 Real-time quantitative PCR analysis of the relative gene expression of embryogenic marker genes (LEC1, LEC2 and FUS3) and the wound-induced transcription factor (WINDI) in untreated (TIBA-) and TIBA-treated (TIBA+) seedling roots and in untreated root explants cultured for 4 days on the ARM IIr medium followed by a hormone-free culture for 5 days. Expression in untreated seedling root was used as reference. The expression of the AT2G41960 gene

1998) as well as for proper root responses to localized supply of nitrate (Guo et al. 2005). Inhibition of shoot-to-root auxin transport and lateral root primordium formation might promote regeneration competence altering the root's responses to exogenous hormones.

Acknowledgements Open access funding provided by MTA Biological Research Center (MTA SZBK). The presented work has been implemented with the support provided from the National Research, Development and Innovation Fund of Hungary, financed under the project no. NKFI-6 K 108802.

Author contributions Dóra Bernula: Investigation, Writing-review and editing; Péter Benkő: Investigation, Methodology; Nikolett Kaszler: Methodology; Ildikó Domonkos: Methodology; Ildikó Valkai: Investigation; Réka Szőllősi: Methodology; Györgyi Ferenc: Methodology; Ferhan Ayaydin: Methodology; Visualization; Attila Fehér: Conceptualization; Funding acquisition; Writing — original draft; Project administration; Katalin Gémes: Data curation; Formal analysis; Writing—review and editing; Supervision.

\section{Compliance with ethical standards}

Conflict of interest We declare that all authors viewed the manuscript and agreed in the submission to Plant Cell, Tissue, and Organ Culture and they have no conflict of interest to be reported.

Open Access This article is distributed under the terms of the Creative Commons Attribution 4.0 International License (http://creativeco mmons.org/licenses/by/4.0/), which permits unrestricted use, distribution, and reproduction in any medium, provided you give appropriate credit to the original author(s) and the source, provide a link to the Creative Commons license, and indicate if changes were made.

\section{References}

Bai B, Su YH, Yuan J, Zhang XS (2013) Induction of somatic embryos in Arabidopsis requires local YUCCA expression mediated by the down-regulation of ethylene biosynthesis. Mol Plant 6:12471260. https://doi.org/10.1093/mp/sss 154

Bhojwani SS, Dantu PK (2013) Plant tissue culture: an introductory text. Springer, New Delhi. https://doi.org/10.1007/978-81-322-1026-9

Bronner R, Jeannin G, Hahne G (1994) Early cellular events during organogenesis and somatic embryogenesis induced on immature zygotic embryos of sunflower (Helianthus annuus). Can J Bot 72:239-248. https://doi.org/10.1139/b94-032

Casson SA, Lindsey K, Sim S et al (2006) The turnip mutant of Arabidopsis reveals that LEAFY COTYLEDON1 expression mediates the effects of auxin and sugars to promote embryonic cell identity. Plant Physiol 142:526-541. https://doi.org/10.1104/pp.106.08089 5

Catterou M, Dubois F, Smets R, Vaniet S, Kichey T, Van Onckelen H, Sangwan-Norreel BS, Sangwan RS (2002) hoc: an Arabidopsis mutant overproducing cytokinins and expressing high in vitro 
organogenic capacity. Plant J 30:273-287. https://doi.org/10.1046/ j.1365-313X.2002.01286.x

Charrière F, Hahne G (1998) Induction of embryogenesis versus caulogenesis on in vitro cultured sunflower (Helianthus annuus L.) immature zygotic embryos: role of plant growth regulators. Plant Sci 137:63-71. https://doi.org/10.1016/S0168-9452(98)00128-9

Charrière F, Sotta B, Miginiac É, Hahne G (1999) Induction of adventitious shoots or somatic embryos on in vitro cultured zygotic embryos of Helianthus annuus: variation of endogenous hormone levels. Plant Physiol Biochem 37:751-757. https://doi. org/10.1016/S0981-9428(00)86688-7

Chatfield SP, Capron R, Severino A et al (2013) Incipient stem cell niche conversion in tissue culture: using a systems approach to probe early events in WUSCHEL-dependent conversion of lateral root primordia into shoot meristems. Plant J 73:798-813. https:// doi.org/10.1111/tpj.12085

Chaudhury AM, Letham S, Craig S, Dennis ES (1993) amp1- a mutant with high cytokinin levels and altered embryonic pattern, faster vegetative growth, constitutive photomorphogenesis and precocious flowering. Plant J 4:907-916. https://doi.org/10.1046/ j.1365-313X.1993.04060907.x

Chen JT, Chang WC (2004) TIBA affects the induction of direct somatic embryogenesis from leaf explants of Oncidium. Plant Cell Tissue Organ Cult 79:315-320. https://doi.org/10.1007/s1124 0-004-4613-5

Cheng ZJ, Wang L, Sun W et al (2013) Pattern of auxin and cytokinin responses for shoot meristem induction results from the regulation of cytokinin biosynthesis by AUXIN RESPONSE FACTOR3. Plant Physiol 161:240-251. https://doi.org/10.1104/pp.112.20316 6

Chung H-H, Chen J-T, Chang W-C (2005) Cytokinins induce direct somatic embryogenesis of Dendrobium chiengmai pink and subsequent plant regeneration. In Vitro Cell Dev Biol 41:765-769. https://doi.org/10.1079/IVP2005702

Elhiti M, Stasolla C (2011) Ectopic expression of the Brassica SHOOTMERISTEMLESS attenuates the deleterious effects of the auxin transport inhibitor TIBA on somatic embryo number and morphology. Plant Sci 180:383-390. https://doi.org/10.1016/j.plant sci.2010.10.014

Fehér A (2015) Somatic embryogenesis-stress-induced remodeling of plant cell fate. Biochim Biophys Acta-Gene Regul Mech 1849:385-402. https://doi.org/10.1016/j.bbagrm.2014.07.005

Fehér A, Bernula D, Katalin G (2016) The many ways of somatic embryo initiation. In: Loyola-Vargas VM, Ochoa-Alejo N (eds) Somatic embryogenesis: fundamental aspects and applications. Springer, Cham, pp 23-37. https://doi.org/10.1007/978-3-31933705-0_3

Feldmann KA, David Marks M (1986) Rapid and efficient regeneration of plants from explants of Arabidopsis thaliana. Plant Sci 47:63-69. https://doi.org/10.1016/0168-9452(86)90011-7

Gaj MD (2001) Direct somatic embryogenesis as a rapid and efficient system for in vitro regeneration of Arabidopsis thaliana. Plant Cell Tissue Org Cult 64:39-46. https://doi.org/10.1023/A:10106 79614721

Gaj MD (2004) Factors influencing somatic embryogenesis induction and plant regeneration with particular reference to Arabidopsis thaliana (L.) Heynh. Plant Growth Regul 43:27-47. https://doi. org/10.1023/B:GROW.0000038275.29262.fb

Gaj MD, Zhang S, Harada JJ, Lemaux PG (2005) Leafy cotyledon genes are essential for induction of somatic embryogenesis of Arabidopsis. Planta 222:977-988. https://doi.org/10.1007/s0042 5-005-0041-y

Grzyb M, Kalandyk A, Mikuła A (2018) Effect of TIBA, fluridone and salicylic acid on somatic embryogenesis and endogenous hormone and sugar contents in the tree fern Cyathea delgadii Sternb. Acta Physiol Plant 40:1. https://doi.org/10.1007/s11738-017-2577-4
Guo Y, Chen F, Zhang F, Mi G (2005) Auxin transport from shoot to root is involved in the response of lateral root growth to localized supply of nitrate in maize. Plant Sci 169:894-900. https://doi. org/10.1016/j.plantsci.2005.06.007

Horstman A, Bemer M, Boutilier K (2017) A transcriptional view on somatic embryogenesis. Regeneration 4:201-216. https://doi. org/10.1002/reg2.91

Ikeda M, Ohme-Takagi M (2014) TCPs, WUSs, and WINDs: families of transcription factors that regulate shoot meristem formation, stem cell maintenance, and somatic cell differentiation. Front Plant Sci 5:3-6. https://doi.org/10.3389/fpls.2014.00427

Ikeuchi M, Ogawa Y, Iwase A, Sugimoto K (2016) Plant regeneration: cellular origins and molecular mechanisms. Development 143:1442-1451. https://doi.org/10.1242/dev.134668

Iwase A, Mitsuda N, Koyama T et al (2011a) The AP2/ERF transcription factor WIND1 controls cell dedifferentiation in Arabidopsis. Curr Biol 21:508-514. https://doi.org/10.1016/j.cub.2011.02.020

Iwase A, Ohme-Takagi M, Sugimoto K (2011b) WIND1 A key molecular switch for plant cell dedifferentiation. Plant Signal Behav 6:1943-1945. https://doi.org/10.4161/psb.6.12.18266

Iwase A, Mita K, Nonaka S et al (2015) WIND1-based acquisition of regeneration competency in Arabidopsis and rapeseed. J Plant Res 128:389-397. https://doi.org/10.1007/s10265-015-0714-y

Iwase A, Harashima H, Ikeuchi M et al (2017) WIND1 promotes shoot regeneration through transcriptional activation of ENHANCER OF SHOOT REGENERATION1 in Arabidopsis. Plant Cell 29:54-69. https://doi.org/10.1105/tpc.16.00623

Jiménez VM (2005) Involvement of plant hormones and plant growth regulators on in vitro somatic embryogenesis. Plant Growth Regul 47:91-110. https://doi.org/10.1007/s10725-005-3478-x

Kakimoto T (2001) Identification of plant cytokinin biosynthetic enzymes as dimethylallyl diphosphate: ATP/ADP isopentenyltransferases. Plant Cell Physiol 42:677-685. https://doi. org/10.1093/pcp/pce112

Kikuchi A, Sanuki N, Higashi K, Koshiba T, Kamada H (2006) Abscisic acid and stress treatment are essential for the acquisition of embryogenic competence by carrot somatic cells. Planta 223:637645. https://doi.org/10.1007/s00425-005-0114-y

Ledwoń A, Gaj MD (2011) LEAFY COTYLEDON1, FUSCA3 expression and auxin treatment in relation to somatic embryogenesis induction in Arabidopsis. Plant Growth Regul 65:157-167. https ://doi.org/10.1007/s10725-011-9585-y

Lotan T, Ohto M, Yee KM et al (1998) Arabidopsis LEAFY COTYLEDON1 is sufficient to induce embryo development in vegetative cells. Cell 93:1195-1205. https://doi.org/10.1016/S0092 -8674(00)81463-4

Mantiri FR, Kurdyukov S, Lohar DP, Sharopova N, Saeed NA, Wang XD et al (2008) The transcription factor MtSERF1 of the ERF subfamily identified by transcriptional profiling is required for somatic embryogenesis induced by auxin plus cytokinin in Medicago truncatula. Plant Physiol 146:1622-1636. https://doi. org/10.1104/pp.107.110379

Márton L, Browse J (1991) Facile transformation of Arabidopsis. Plant Cell Rep 10:235-239. https://doi.org/10.1007/BF00232565

Meinke DW, Franzmann LH, Nickle TC, Yeung EC (1994) Leafy cotyledon mutants of Arabidopsis. Plant Cell 6:1049-1064. https://doi. org/10.1105/tpc.6.8.1049

Mujib A, Ali M, Tonk D et al (2016) Embryogenesis in ornamental monocots: plant growth regulators as signalling element. In: Mujib A (ed) Somatic embryogenesis in ornamentals and its applications. Springer, New Delhi, pp 187-201. https://doi. org/10.1007/978-81-322-2683-3_12

Nishiwaki M, Fujino K, Koda Y, Masuda K, Kikuta Y (2000) Somatic embryogenesis induced by the simple application of abscisic acid to carrot (Daucus carota L.) seedlings in culture. Planta 211:756759. https://doi.org/10.1007/s004250000387 
Pernisová M, Kuderová A, Hejátko J (2011) Cytokinin and auxin interactions in plant development: metabolism, signalling, transport and gene expression. Curr Protein Pept Sci 12:137-147. https:// doi.org/10.2174/138920311795684887

Reed RC, Brady SR, Muday GK (1998) Inhibition of auxin movement from the shoot into the root inhibits lateral root development in Arabidopsis. Plant Physiol 118:1369-1378. https://doi. org/10.1104/pp.118.4.1369

Reynolds TL (1986) Somatic embryogenesis and organogenesis from callus cultures of Solanum carolinense. Am J Bot 73:914-918. https://doi.org/10.1002/j.1537-2197.1986.tb12131.x

Rosspopoff O, Chelysheva L, Saffar J et al (2017) Direct conversion of root primordium into shoot meristem relies on timing of stem cell niche development. Development 144:1187-1200. https://doi. org/10.1242/dev. 142570

Sagare AP, Lee YL, Lin TC et al (2000) Cytokinin-induced somatic embryogenesis and plant regeneration in Corydalis yanhusuo (Fumariaceae)—a medicinal plant. Plant Sci 160:139-147. https ://doi.org/10.1016/S0168-9452(00)00377-0

Schaller GE, Bishopp A, Kieber JJ (2015) The yin-yang of hormones: cytokinin and auxin interactions in plant development. Plant Cell 27:44-63. https://doi.org/10.1105/tpc.114.133595

Schiavone FM, Cooke TJ (1987) Unusual patterns of somatic embryogenesis in the domesticated carrot: developmental effects of exogenous auxins and auxin transport inhibitors. Cell Differ 21:53-62. https://doi.org/10.1016/0045-6039(87)90448-9

Scholl RL, May ST, Ware DH (2000) Seed and molecular resources for Arabidopsis. Plant Physiol 124:1477-1480. https://doi. org/10.1104/pp.124.4.1477

Skoog F, Miller CO (1957) Chemical regulation of growth and organ formation in plant tissues cultured in vitro. Symp Soc Exp Biol 11:118-130

Stone SL, Kwong LW, Yee KM et al (2001) LEAFY COTYLEDON2 encodes a B3 domain transcription factor that induces embryo development. Proc Natl Acad Sci USA 98:11806-11811. https:// doi.org/10.1073/pnas.201413498

Su YH, Zhang XS (2014) The hormonal control of regeneration in plants. Curr Top Dev Biol 108:35-69. https://doi.org/10.1016/ B978-0-12-391498-9.00010-3
Su YH, Zhao XY, Liu YB et al (2009) Auxin-induced WUS expression is essential for embryonic stem cell renewal during somatic embryogenesis in Arabidopsis. Plant J 59:448-460. https://doi. org/10.1111/j.1365-313X.2009.03880.X

Su YH, Su YX, Liu YG, Zhang XS (2013) Abscisic acid is required for somatic embryo initiation through mediating spatial auxin response in Arabidopsis. Plant Growth Regul 69:167-176. https ://doi.org/10.1007/s10725-012-9759-2

Su YH, Liu YB, Bai B, Zhang XS (2015) Establishment of embryonic shoot-root axis is involved in auxin and cytokinin response during Arabidopsis somatic embryogenesis. Front Plant Sci 5:792. https ://doi.org/10.3389/fpls.2014.00792

Sugiyama M (2015) Historical review of research on plant cell dedifferentiation. J Plant Res 128:349-359. https://doi.org/10.1007/ s10265-015-0706-y

Sun J, Niu Q-W, Tarkowski P, Zheng B, Tarkowska D, Sandberg G et al (2003) The Arabidopsis AtIPT8/PGA22 gene encodes an isopentenyl transferase that is involved in de novo cytokinin biosynthesis. Plant Physiol 131:167-176. https://doi.org/10.1104/pp.011494

Valvekens D, Van Montagu M, Van Lijsebettens M (1988) Agrobacterium tumefaciens-mediated transformation of Arabidopsis thaliana root explants by using kanamycin selection. Proc Natl Acad Sci USA 85:5536-5540. https://doi.org/10.1073/pnas.85.15.5536

Winter D, Vinegar B, Nahal H et al (2007) An "electronic fluorescent pictograph" browser for exploring and analyzing large-scale biological data sets. PLoS ONE 2:e718. https://doi.org/10.1371/journ al.pone. 0000718

Xu L, Huang H (2014) Genetic and epigenetic controls of plant regeneration. Curr Top Dev Biol 108:1-33. https://doi.org/10.1016/ B978-0-12-391498-9.00009-7

Yumbla-Orbes M, da Cruz ACF, Pinheiro MVM et al (2017) Somatic embryogenesis and de novo shoot organogenesis can be alternatively induced by reactivating pericycle cells in Lisianthus (Eustoma grandiflorum (Raf) Shinners) root explants. In Vitro Cell Dev Biol 53:209-218. https://doi.org/10.1007/s11627-017-9800-2

Publisher's Note Springer Nature remains neutral with regard to jurisdictional claims in published maps and institutional affiliations. 